\title{
Speech Enhancement of Singing Support System
}

\section{for Lartngectomies}

\author{
Hirotaka Nogami $^{\mathrm{a}}$, Yoshihisa Nakatoh ${ }^{\mathrm{a}^{*}}$, Kenji Matsui $^{\mathrm{b}}$ \\ ${ }^{a}$ Kyushu Institute of Technology, 1-1 Sensui-cho, Tobata-ku, Kitakyusyu 804-8550, Japan \\ ${ }^{\mathrm{b}}$ Osaka Institute of Technology, 5-16-1 Omiya, Asahi-ku,Osaka 535-0002, Japan \\ *Corresponding Author: nakatoh@ecs.kyutech.ac.jp
}

\begin{abstract}
This research, purpose to develop a singing support system for laryngectomies, we examined a singing sound source along with a sound quality improvement of the processed singing voice. For the singing sound source, the Bark LPC residual signal is obtained by flattening the LPC residual signal with Bark spectrum. To improve the sound quality of singing voice, SS method which is one of speech enhancement methods is used. We carry out listening experiments for the two singing voices, "singing voice before processing" and "singing voice after processing". We evaluate "Intelligible", "Noisy" and "Natural" characteristics between them. As for the "Intelligible" and "Noisy", improvements are observed for all phonemes. As for "Natural", there changes phonemes of /a/, /i/ and /o/, but there are significantly improved for /u/ and /e/.
\end{abstract}

Keywords: LPC residual signal, Electric-Larynx, Singing Syetem, Bark spectrum

\section{Introduction}

Singing a song is the major forms of entertainment, so a lot of people are enjoying it. However, some people cannot produce a natural sound because of handicaps. Laryngectomy is a common technology for treatment of laryngeal cancer. As a result of the treatment, the patient's larynx has to be removed completely. Therefore, although the movement of the mouth and tongue is normal, it cannot vocalize because the vocal cord cannot be vibrated.

The population of laryngectomees is estimated to be more than 30,000 in Japan. Because Electric-Larynx (EL) can be easily obtained, the demand of it is increasing as an alternative vocalization among elderly laryngectomies ${ }^{(1)}$. Also, EL is attached to sing function called Song-mode ${ }^{(2)}$. However, the singing voice of Song-mode is unnatural and
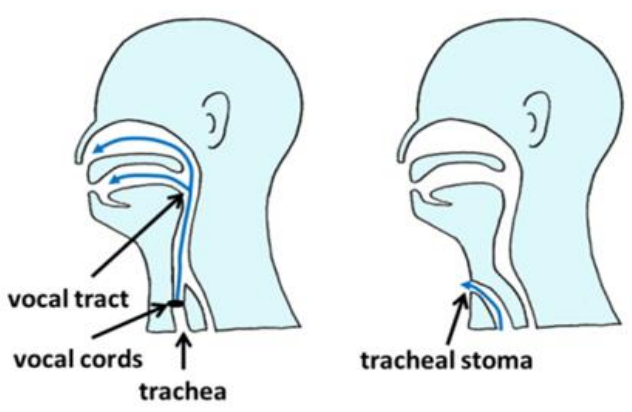

Fig. 1. The route of the air flowing from the lungs. The left figure shows the case of non-handicapped people. The right figure shows the case of the laryngectomies.

cannot fully enjoy singing.

We proposed a singing system that transfers vocal code sound information (pseudo vocal sound source) extracted from real singing voice into the throat by using a vibration speaker ${ }^{(3)}$. In this research, we propose improvement of sound quality to remove the pseudo vocal sounds included in singing voice. Spectral Subtraction method (SS method) is used as a method of sound quality improvement. Sound quality improvement is performed using the SS method, and they are evaluated for Noisy, Intelligible and Natural.

\section{Proposed Singing Support System for Laryngectomies}

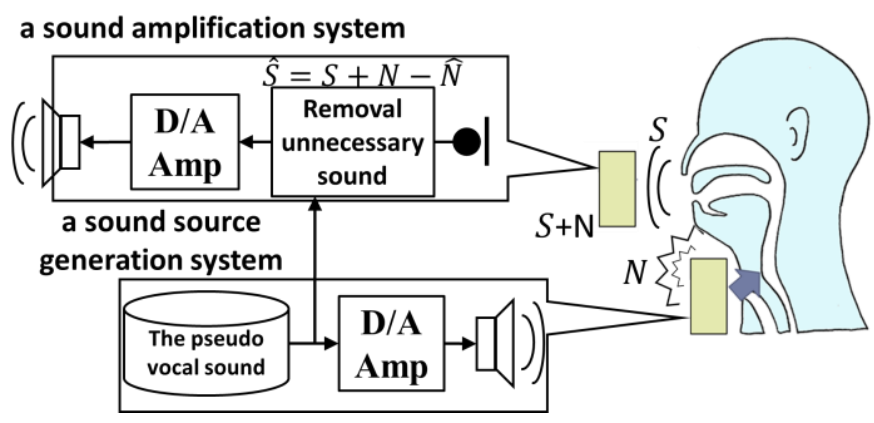

Fig. 2. System configuration (C) 2017 The Institute of Industrial Applications Engineers, Japan. 


\subsection{Summary of the Singing Support System}

Figure 2 is a singing support system for laryngectomies. It consists of a sound amplification system and a sound source generation system. Beforehand, the pseudo vocal sound source extracted and processed in advance from the singing voice is stored in the database. We output it from the vibration speaker and transmit it inside the throat to sing. We pick up the singing voice with a microphone. At that time, the pseudo vocal sound source used is transmitted through the air and mixed in the microphone. We improve the sound quality of the singing voice by removing the unnecessary components. Enjoy singing be sending this processed sound from the speaker.

\subsection{Sound Source used for the System}

Figure 3 is a method of creating a pseudo vocal sound source used for the system. This method consists four key parts:

(1) Time frequency transformation (DCT(Discrete Cosine Transformation))

(2) LPC (Linear Predictive Cording) analysis

(3) Calculation of Bark Spectrum

(4) Flattening of DCT value by the spectral bark scale

Input signal $(\mathrm{x}(\mathrm{t}))$ is singing voice of singer who recorded in advance. To convert frequency $(X(\omega))$ of input signal. Also, to separate input signal in vocal cord and vocal tract, input signal is transformed LPC spectrum envelope $(\mathrm{F}(\omega))$ by LPC analysis block. Therefore, inverse filtering $F(\omega)$ on $X(\omega)$ obtains LPC residual signal. Further, the bark LPC residual signal $(\mathrm{G}(\omega))$ is obtained by flattening the DCT coefficient with the Bark spectrum $(\mathrm{B}(\omega))$ of the LPC residual signal $(\{\mathrm{X}(\omega) / \mathrm{F}(\omega)\} / \mathrm{B}(\omega))$. $\mathrm{g}(\mathrm{t})$ is obtained by IDCT. This bark LPC residual signal is used as a pseudo

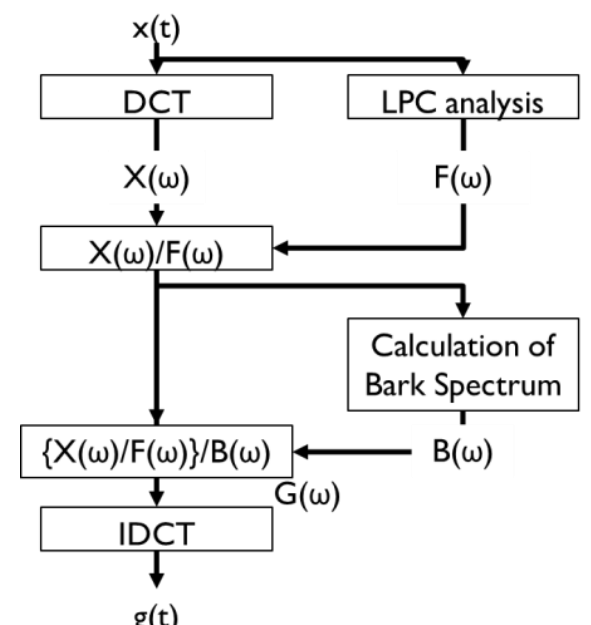

Fig. 3. Bark LPC residual wave processing flow vocal sound source of the system. This sound sampling frequency is $16 \mathrm{kHz}$, LPC analysis order is 16 dimensions.

Bark spectrum is a spectrum envelope that can be calculated based on the Bark scale derived from the observation of the critical bandwidth with respect to the frequency resolution of auditory sense. A nonlinear frequency axis elongation / shrinkage scale and corresponds to 24 critical bands of hearing. 24 critical bands of hearing are $\{0,100,200,300,400,510,630,770,920,1080,1270$, 1480, 1720, 2000, 2320, 2700, 3150, 3700, 4400, 5300, $6400,7700,9500,12000,15500[\mathrm{~Hz}]\}^{(4)}$. In order not to remove the fundamental frequency, Bark spectrum is not applied below $300[\mathrm{~Hz}]$.

\subsection{Unnecessary components removal by SS method}

When singed by a vibration speaker, the vibration sound emitted from the vibration speaker also mixes in the microphone. We propose a method to remove this unnecessary components $(\mathrm{N}(\omega, \mathrm{t}))$ moment by SS method ${ }^{(5)}$. SS method is a type of speech enhancement method performed in the frequency domain. In this research, the unnecessary components mixed into the microphone is considered as a pseudo vocal sound source used for singing. Therefore, the unnecessary components are removed with a pseudo vocal sound source (Bark LPC residual signal) by using singing. To subtract Bark LPC residual signal $(\widehat{G}(\omega, t))$ at the same time from singing voice $(\mathrm{S}(\omega, \mathrm{t})+\mathrm{N}(\omega$, t)) by the following equation. In this case, $\alpha$ is a Spectral Subtraction coefficient (SS coefficient).

$$
|\hat{S}(\omega, t)|=|S(\omega, t)+N(\omega, t)|-\alpha|\widehat{G}(\omega, \mathrm{t})|
$$

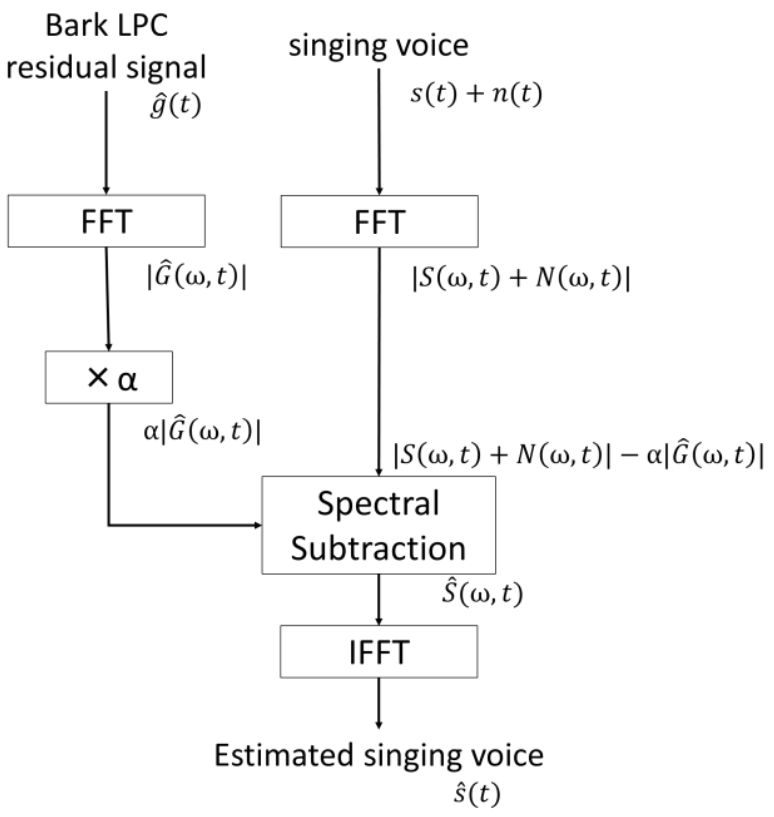

Fig. 4. Spectral Subtraction method flow 


\section{Evaluation experiment}

\subsection{Evaluation methods}

In this experiment, we use a singing voice using a pseudo vocal sound source. We output the pseudo-vocal sound source through a vibration speaker, and sing by pressing it against the throat. Figure 5 is the photograph of the vibration speaker. We use a VR 3000, KOBATEL vibration speaker.

Experimental conditions are shown in Table.1. "Twinkle Twinkle Little Star" of nursery rhymes which all vowels are included in lyrics is selected. Syllables including five vowels extracted from (1) kirakirahikaru, osoranohoshiyo, (3) mabatakishiteha and (4) minnawomiteru from "Twinkle Twinkle Little Star" are evaluated by data cut out. We carry out listening

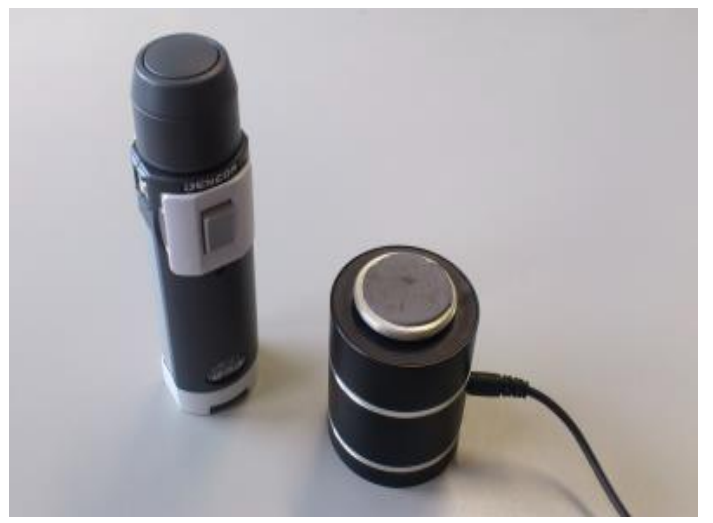

Fig. 5 The left figure shows EL and the right figure shows the vibration speaker.

Table. 1 Experimental conditions

\begin{tabular}{|c|c|}
\hline Used sound & $\begin{array}{ll}\text { A: Original singing voice } & \\
\text { B: Processing singing voice with } & \alpha=0.6 \\
\text { C: Processing singing voice with } & \alpha=1.0 \\
\text { D: Processing singing voice with } & \alpha=1.4 \\
\text { E: Processing singing voice with } & \alpha=1.8\end{array}$ \\
\hline $\begin{array}{l}\text { Used song and } \\
\text { used part }\end{array}$ & $\begin{array}{l}\text { Song:Twinkle Twinkle Little Star } \\
\text { (1) Kirakirahikaru } \\
\text { (2) Osoranohosiyo } \\
\text { (3) Mabatakishiteha } \\
\text { (4) minnawomiteru }\end{array}$ \\
\hline Evaluation word & $\begin{array}{ll}\text { (1) } & \text { Noisy } \\
\text { (2) } & \text { Intelligible } \\
\text { (3) } & \text { Natural }\end{array}$ \\
\hline Singers & One person(normal person) \\
\hline subjects & Seven people(normal person) \\
\hline Number of run & Five times(The first time is practice.) \\
\hline
\end{tabular}

experiments for the two singing voices, "singing voice before processing" and "singing voice after processing". We use a variant of the Nakaya pair-wise comparison of Scheffe to analyze the results ${ }^{(6)}$. We evaluate "Noisy", "Intelligible" and "Natural" and take the average. We regard the sound before processing as A, and let the sound after processing be $\mathrm{B}, \mathrm{C}, \mathrm{D}$ and $\mathrm{E}$ for each SS coefficient (B:0.6, C:1.0, D:1.4, E:1.8). The type of choices in this evaluation consist of five grades (-2: sound A is very good, -1: sound A is good, 0: A and B are same level, 1: sound B is good, 2: sound B is very good). Figure 6 is a screen of listening experiment. The subjects consist of seven healthy individuals.

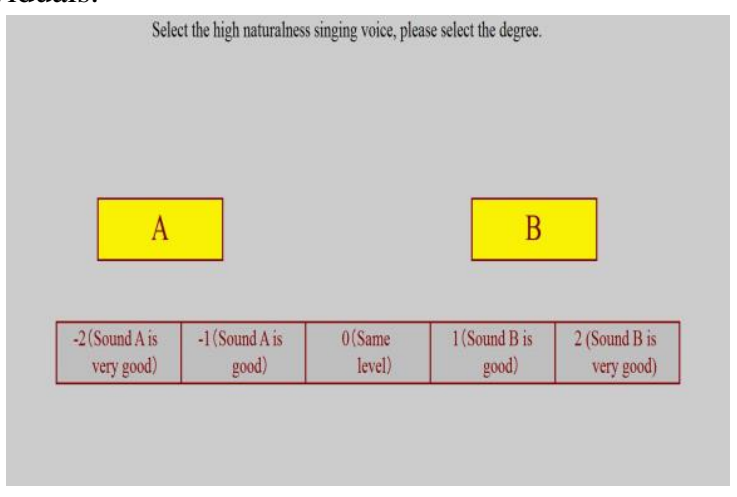

Fig. 6 A screen of listening experiment.

\subsection{The result of evaluation experiment}

Listening experiments result for each evaluation word are shown. For each vowel comparison, the results for "Noisy" are shown in figure 7, the results for "Intelligible" are shown in figure 8, and the results for "Natural" are shown in figure 9. The vertical axis is the average preference level of the singing voice after processing and the singing voice before processing. The horizontal axis shows the significance of the SS coefficient, and the line is the $99 \%$ confidence interval. A positive value indicates singing voice after processing, and a negative value indicates that singing voice before processing is significant.

As for "Noisy", it can be seen that the noise feeling is alleviated as the SS coefficient increases in all phonemes.

As for "Intelligible", it can be seen that there improved in all phonemes by using the SS method. Especially for $/ \mathrm{a} /$ and $/ \mathrm{o} /$, it appeared greatly. On the other hand, almost no difference in "Intelligible" is observed in the SS coefficient range of 0.6 to 1.8 .

As for "Natural", /a/, /i/ and /o/, are significantly improved. On the other hand, no significance is found for /u/ and /e/. 
As for "Noisy" and "Intelligible", all phonemes in all SS coefficients improved. As for "Natural", we can improve /a/, /i/ and /o/. On the other hand, /u/ and /e/ did not show the significance.

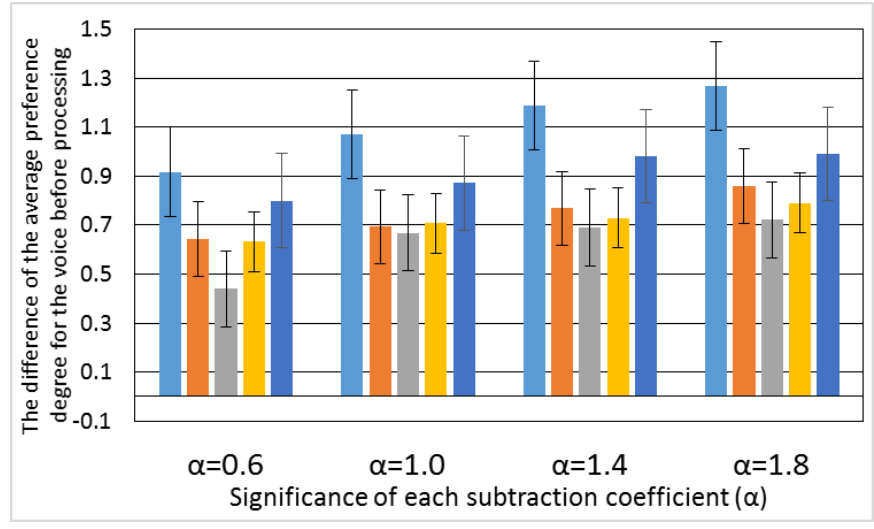

Fig. 7 Comparison of "Noisy" before and after processing

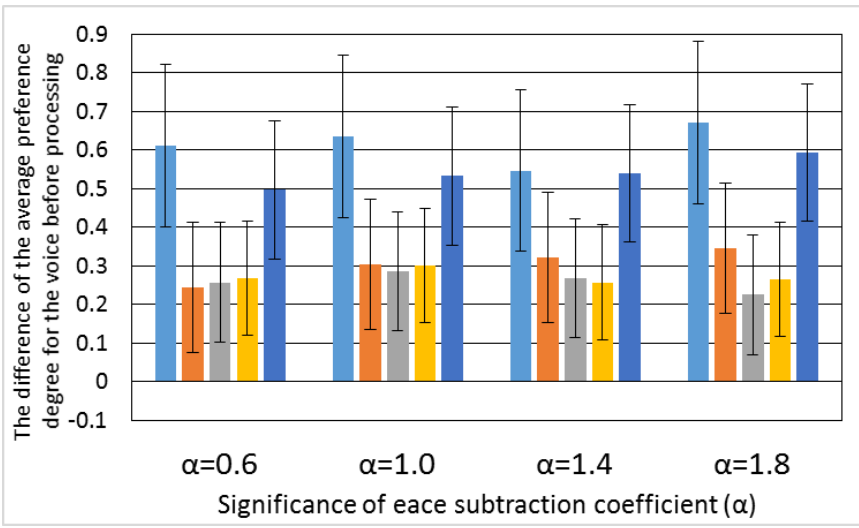

Fig. 8 Comparison of "Intelligible" before and after processing

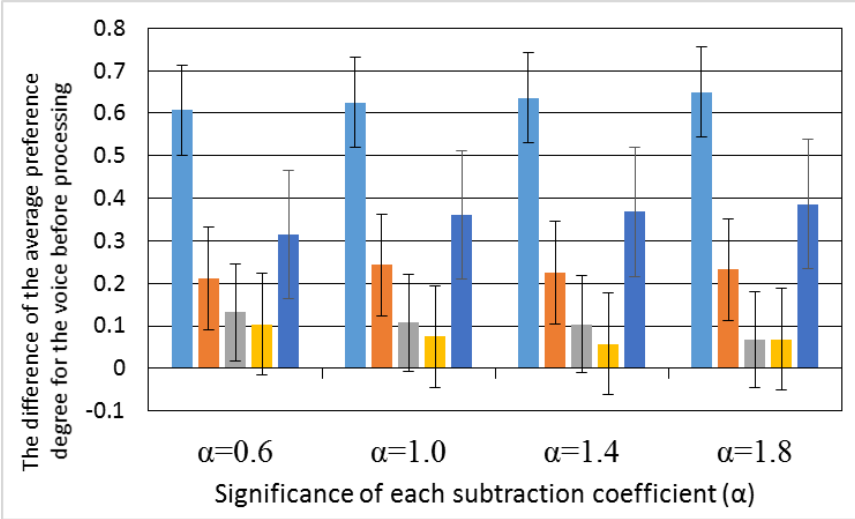

Fig. 9 Comparison of "Natural" before and after processing

\section{Conclusions}

In this research, sound quality improvement is performed by removing the unnecessary components by the pseudo vocal sound source which generated by using the SS method in the singing support system for the laryngectomies. By removing unnecessary components, the improvement observed on all phonemes of "Noisy" and "Intelligible" improved obviously. As for "Natural", we can improve /a/, /i/ and /o/. On the other hand, /u/ and /e/ did not show the significance. In the future, we will study the influence on the vocal character by the different singers, and further study the improvement of the sound quality.

\section{Acknowledgment}

This work was partially supported by a Grant-inAid for Science Research (No.15K01487) from the Japan Society for the Promotion of Science.

\section{References}

(1) Hiroyuki Fukuda, "Today and tomorrow of alaryngeal voice with an artificial larynx", Journal of the Acoustical Society of Japan Vol.44 pp.130-134(1988).

(2) Takashi Himeno, YutoTnaka, and Yoshihisa Nakatoh: "Sound Quality Improvement of Singing Voice by Electro-Larynx", Proceedings of IIAE Annual Conference 2014, September 28, 2014, pp.16-17(2014), DOI: 10.12792/iiae2014.009

(3) T.Himeno et al.Singing support system using LPC residual wave for laryngectomee, IEEE International Conference on Consumer Electronics(ICCE), pages395-396, 2016

(4) Smith JO, Abel JS: "Bark and ERB bilinear transforms", IEEE Transactions on Speech and Audio Processing, pp697-708, 1999

(5) M. Berouti, R. Schwartz, and J. Makhoul, "Enhancement of speech corrupted by acoustic noise," in Proc. of the Int. Conf. on Acoustics Speech, and Signal Processing (ICASS)m vol. 4, Apr 1979, pp.208-211.

(6) Sato Makoto : "Tokeiteki kannokensaho StatisticalSensuality Laboratory Procedure", Nikkagiren ( JUSE ), pp.231-270, 1985 